\title{
Crystallography in India: Past, Present and Future
}

\section{Introduction}

On 20 January 2014, while flagging off the celebrations of the year of crystallography in Paris, the Secretary-General of the United Nations stated, "it is at the very core of revealing the structure of DNA, fabricating computer memories and designing potent new drugs, but only a tiny fraction of the world's people are aware of the science of crystallography, a knowledge gap the United Nations hopes to help erase this year."

\section{The past}

The thoughts that come to the mind immediately when the word 'crystal' is mentioned are essentially centered around jewels, purity, faith healing and often future prediction. Indeed, India has a traditional attachment to these aspects from medieval times. The recognition of symmetry in materials has been a part and parcel of our culture and the rich heritage that exists in terms of temple architecture and carvings (figure 1) spread over several centuries.

The concepts of three dimensional objects and the recognition of optical effects to augment features in such objects were documented through centuries. The art of 'Rangavalli or Rangoli' practiced in every household uses the principle of lattice points to generate motifs based on symmetry elements (figure 2).

India's scientific endeavour, as many other developments in the last century, matured with the freedom struggle as a backdrop. It may be claimed that the modern scientific era began in early $20^{\text {th }}$ century. The starting of two centres of higher education, namely, Indian Institute of Science (IISc) at Bangalore and Indian Association for the Cultivation of Science (IACS) at Calcutta, coincided with these efforts. Work in crystallographic research towards the structure determination efforts began with the work of K. Banerjee at IACS with the structures of naphthalene and anthracene being solved (Nature, 125, 456, 1930). Even though considerable interest in crystallographic research existed several years before, the discovery that X-ray diffraction can lead to structure determination catalyzed interest in many scientists including C.V. Raman, K. S. Krishnan, M. N. Saha and S. Bhagavantam to name a few. Crystallography was regarded as the science of condensed matter with emphasis on the atomic and molecular structure and its relation to physical and chemical properties. The analysis was thus primarily considered a job for the physicists initially, and this concept percolated deep in Indian science. Indeed, crystallography was considered a component of physical sciences for several decades until the importance of structure-property relationships was established, which brought in the idea of including chemists and biologists. Interestingly, crystallographers (physicists) were employed in chemistry and biology departments only in the late 1960s.

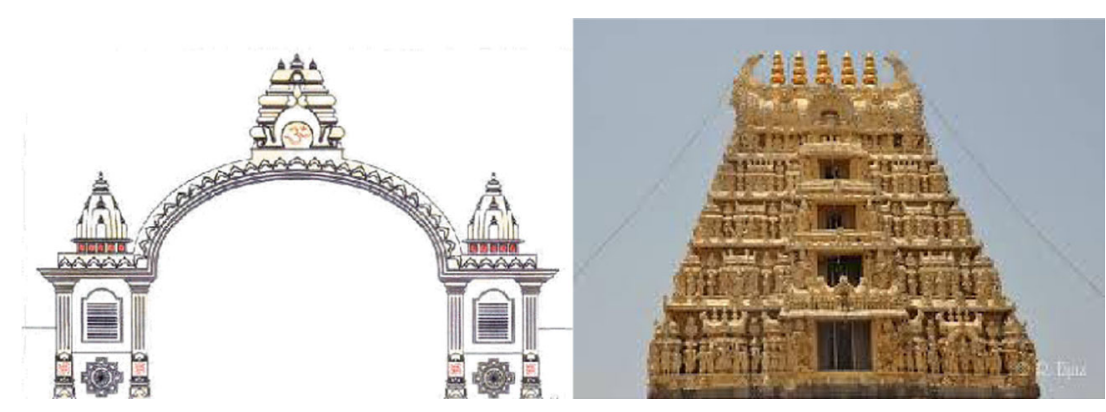

Figure 1. Design of a temple entrance and carvings on the 'Gopuram.' 

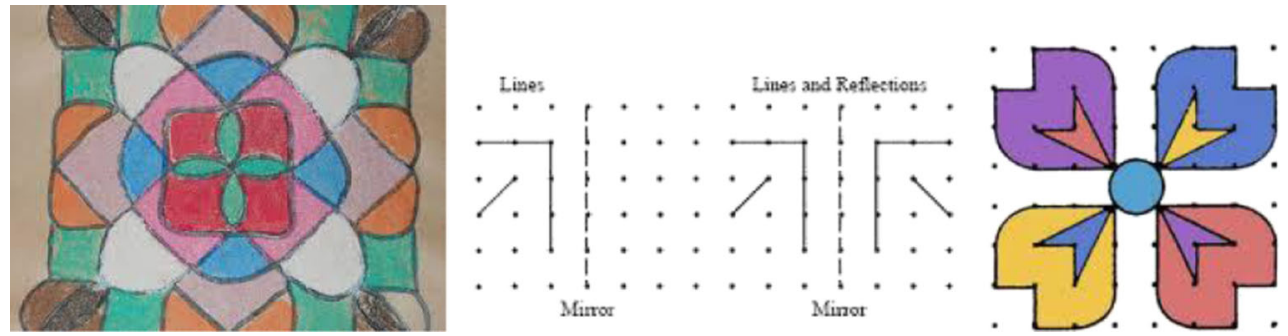

Figure 2. 'Rangoli' and its generation via a lattice concept.

The technological developments percolated to India obviously due to the interactions with the scientists in Great Britain. Preliminary equipments were imported (figure 3), and the interest in structural science began at several centres around the country. Metallurgists and geologists eventually joined this area of research with Banaras Hindu University (BHU) spearheading such activities. It is of interest to note that an X-ray film manufacturing unit was started (INDU films) at Ootacamand (Ooty) and X-ray generators were manufactured at Calcutta (Kolkata) by Radon House Pvt. Ltd., inspired by Siddharth Ray and Gowri Ray in the late 1960s.

C. V. Raman, after moving to Bangalore, provided a major boost with G. N. Ramachandran and S. Ramaseshan carrying out path breaking work in structural science with X-ray diffraction and crystallography as the main experimental modules. They developed strong groups with students like M. A. Viswamitra, K. Venkatesan, R. Chidambaram and H. Manohar. A parallel group developed under the leadership of A. R. Varma at BHU, Varanasi.

G. N. Ramachandran made seminal contributions to the development of X-ray diffraction techniques, and his discoveries were at that time in tandem with several significant strides made around the world including the work of Watson and Crick discovering the double helix in DNA. Indeed, Ramachandran's triple helix in collagen presented much more challenging work in terms of interpreting the fibre diffraction pattern (figure 4). Other major contributions from him include the phi-psi plot depicting the conformational angles in a peptide unit, which constitutes the building block in large proteins and his suggested methods of Fourier synthesis, which provides a basis for structure evaluation.

This is around the time crystallographic research made significant strides and established itself as an unequivocal tool for establishing structures of solids. Work done by Linus Pauling, Dorothy Hodgkins, Watson and Crick, Kathleen Lonsdale and several other leading crystallographers were coming to focus on the importance of X-ray crystallography to the understanding of molecules, chemical bonding, etc., and the basic factors influencing structure-property correlations were getting realized. Ramaseshan and Venkatesan at this juncture came up with the idea of using anomalous scattering (absorption edge) to solve the structure of $\mathrm{KMnO}_{4}$. Ajit Ram Varma at BHU (figure 5) made parallel contributions to the development the crystallography in India in terms of understanding the fundamental aspects of polymorphism, an area which is now a key factor in pharmaceutical research.

At this juncture, crystallographic research started to blossom in the country. R. Srinivasan and S. Parthasarathy at the University of Madras supported by G. N. Ramachandran worked on structure
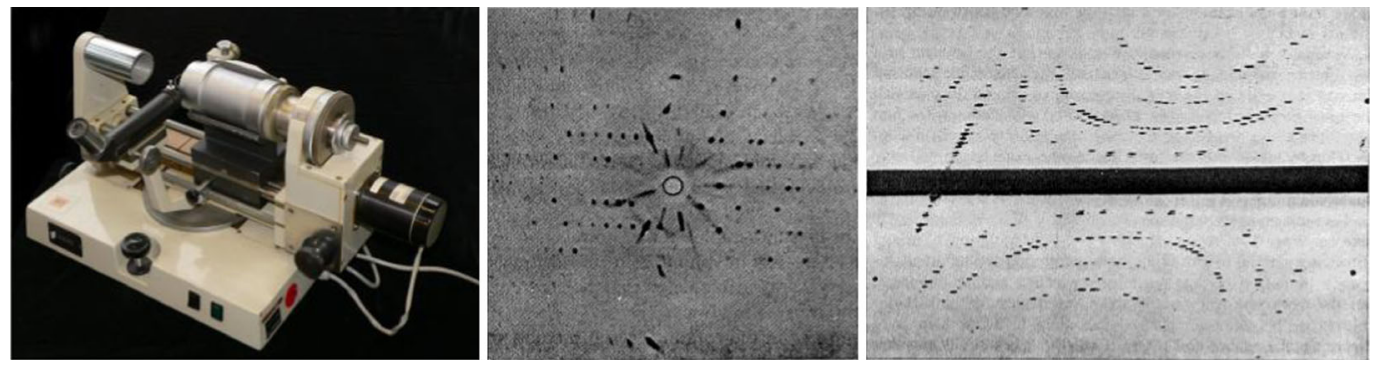

Figure 3. Weissenberg Camera, Rotation and Weisssenberg photographs, which aided characterization. 

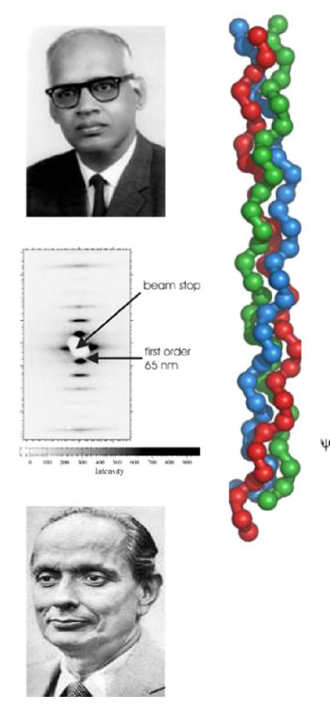

- Triple helix collagen-fibrous proteins (1954).

Fibre diffraction-structural biology in India

- Ramachandran plot $(\phi, \psi)$ for validation of protein structures

- EWALD Prize, 1999

Hard Sphere Ramachandran Map

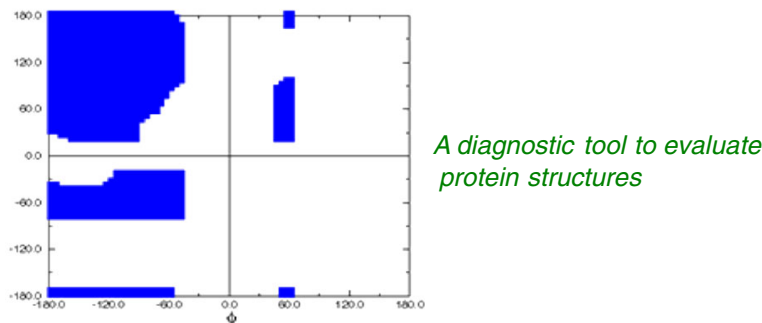

W. L. Bragg, L. Pauling, D. C. Hodgkin, J. D. Bernal, J. M. Bijvoet, N. V. Belov, W. Cochran and A. I. Kitaigorodskii, 1960.

Anomalous dispersion as a structure solution: S. Ramaseshan and K. Venkatesan, 1957.

Basis for MAD and SAD techniques in macromolecular crystallography.

Figure 4. Ramachandran's contributions: structure of collagen triple helix and the phi-psi plot, and Rameseshan's anomalous dispersion interpretation.

determination and refinement strategies. M. A. Viswamitra, H. Manohar and K. Venkatesan initiated extensive work on biological (nucleic acid components, drug design), inorganic compounds (topotactic reactions) and organic chemical crystallography (solid state reactions) at IISc. At the Bhabha Atomic Research Centre (BARC) in Bombay, R. Chidambaram and co-workers began work on hydrogen bonded systems identifying the hydrogen atom positions using neutron diffraction. C.N.R. Rao and his group at IIT Kanpur (later at IISc) determined the structures of a large number of complex oxides using powder diffraction data, leading to solid state chemistry of the modern era. G. B. Mitra at IIT, Kharagpur and L. M. Pant at National Chemical Laboratory, Pune started active research groups in different aspects of
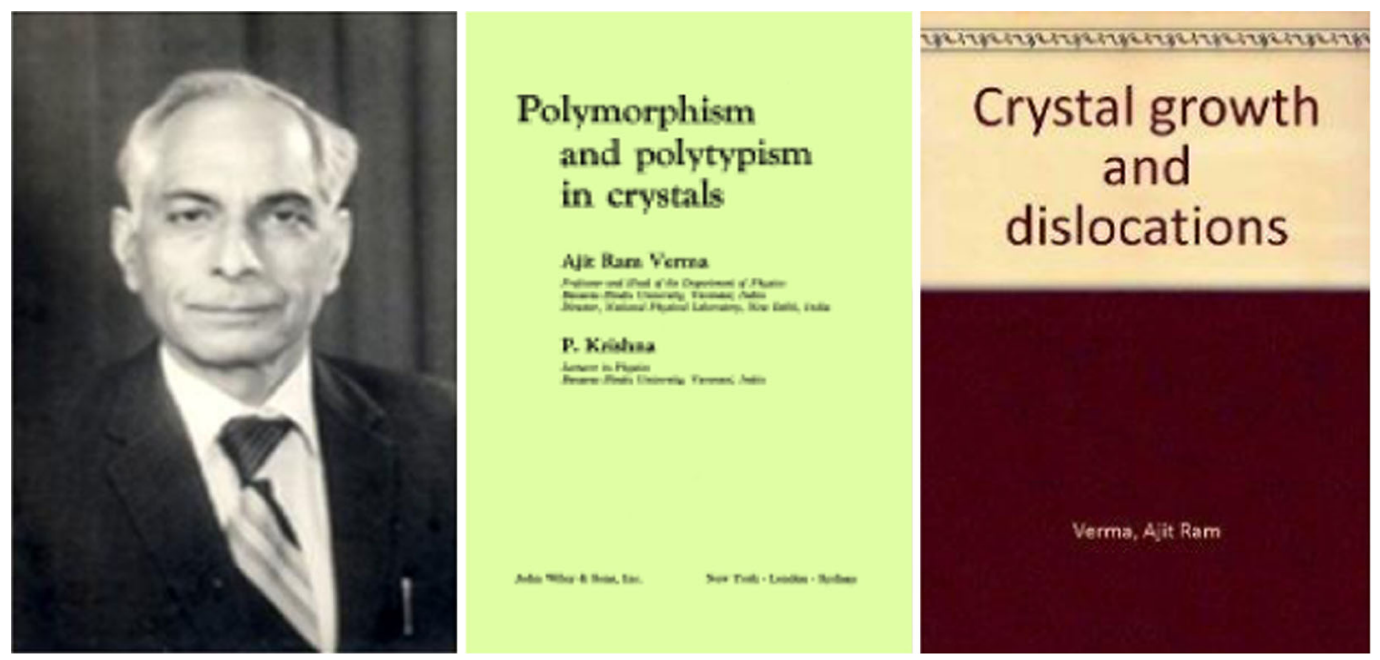

The phenomenon of polytypism is the capacity of a solid to crystallize into more than one modifications, which have essentially the same chemical composition but which differ in the number and the manner of stacking of layers in the unit cell.

Polymorphism is the ability of a solid material to exist in more than one form or crystal structure.

Figure 5. Ajit Ram Varma's contributions to physical crystallography. 
crystallographic research. P. Krishna, K. Lal, O. N. Srivastava, G. Singh and G. C. Trigunayat at BHU continued the tradition of A. R. Varma working on newer aspects of polytypism and polymorphism and obtained useful inputs in physical crystallography. Another group at BHU consisting of T. R. Anantharaman, P. Ramachandra Rao, S. Ranganathan and G. V. S. Sastry worked on areas related to alloys and got some fundamental insights into the five-fold symmetry concepts. Towards the beginning of the 1970s, these groups produced work of international standards. It should be noted that most of them visited different laboratories abroad (mostly in UK) and obtained the required training to carry out such work.

At this time, the western world saw a revolution in technology and fast computers started to emerge. India entered the dark age in crystallographic research (1970-1990). The funding ran abysmally low, and the researchers were left in panic to think of catching up with the developments. The photographic film methods were becoming obsolete and the number of atoms in structures getting solved became unreachable. Diffractometers started mushrooming in UK, Europe and the US, high energy beams became accessible to explore larger structures in quicker time and India started losing in the competition. Several brave hearts (figure 6) took the challenge and started taking bold steps to remain in the race. Collaborations within the country and abroad were set up, all due to the initiatives taken by these stalwarts and each of them started with a bigger set of young researchers. The task was not simple as it was a terrific struggle to cope up with the advances made in data collection technology, high speed computer programs and developments in computer graphics. The choice of problems was getting seriously limited to the existing facilities. I entered the fray as a research student along with four others at this time (figure 7).

Figure 8 gives an idea of the scenario of crystallographic research during the period of 1970 to 1990. Photographic methods for getting data sets involved considerable time and effort and the computers had memories one millionth of the ones we carry now on our mobile phones. Two outstanding scientists, C. N. R. Rao and M. Vijayan (figure 9), realized the difficulties very quickly and put their enormous efforts to get crystallographic research out of the rot. The Government of India was made aware of the status and funds were sought to establish centres to circumvent the deficiencies. The present day research in crystallography hinges delicately on the efforts made by these two individuals, and hereon I shall restrict the description of the current research activities centred around the developments in IISc, in particular, and the spread of the areas to other branches of science where the technique of X-ray diffraction appears strictly as a tool. India, like the other laboratories in the western world has now adopted crystallography as one of the characterization tools (it has become a tool for any researcher and there is no need for expertise).

\section{The Dark Age}

The brave hearts who continued to perform and excel despite lack of state of the art facilities.

- K. Venkatesan with V. Ramamurthy: Photochemical reactions in organic solids

- H. Manohar: Topotactic reactions, inorganic solids and natural products

- M. A. Viswamitra: Nucleic acid crystallography

- R. Chidambaram: Began a program of neutron diffraction in BARC, Mumbai

- K. Lal: Crystal growth and X-ray topography at NPL, New Delhi

- Srinivasan and Parthasarathy: Structure determination techniques, Madras

- L. M. Pant: Charge densities, NCL, Pune

- C. N. R. Rao: Solid state chemistry at IIT Kanpur and later at IISC

- S. Chandrasekhar: Liquid crystals, RRL (Raman Research Laboratory), Bangalore

- T. R. Anantharaman (BHU) and S. Ranganathan (IISC): Quasicrystals
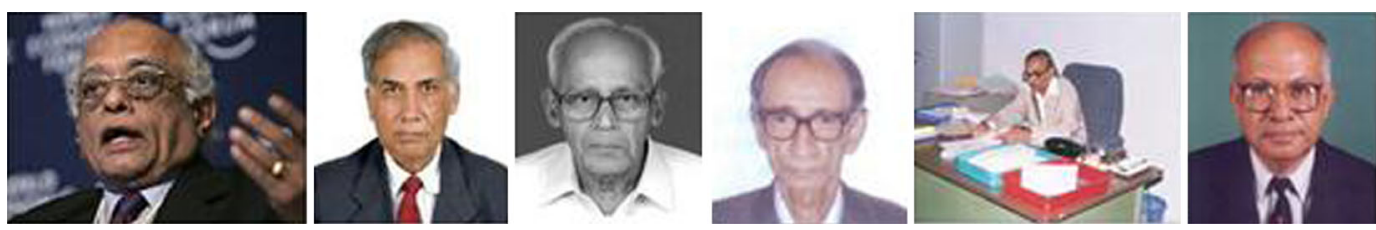

Figure 6. The architects of the current (present) day crystallography. 


\section{The Period of 1970 to 1990 - The Dark Age}

- The struggle to cope up with advances in data collection technology, high speed computers and computer graphics made elsewhere.

- Mostly chose problems to suit the existing facilities.

- I entered the fray along with Ramakumar, M. R. N. Murthy (a year later) (K. Venkatesan) and T. P. Singh and T. N. Bhat (a year later) (M. Vijayan).

- Swaminatha Reddy (M. A. Viswamitra) wrote a program in Fortran IV for block diagonal refinement

- S. K. Sikka: Direct methods structure determination.

- P. Main (Multan) flew into Bangalore to set up the program.
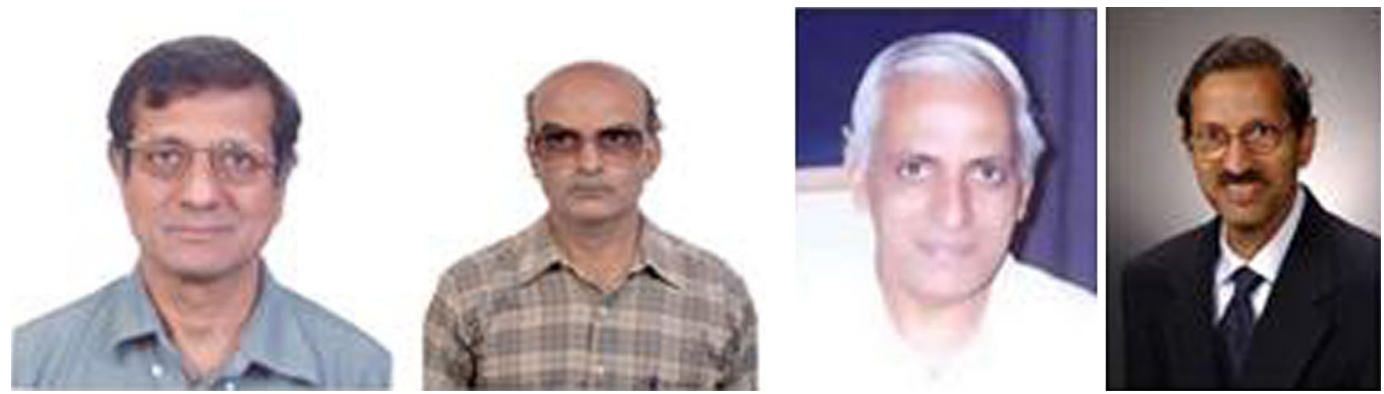

Figure 7. The foundations for the current day structural work were laid during this period by efforts of these young researchers at that time.

\section{The Present}

Thanks to the efforts of the two individuals (C. N. R. Rao and Vijayan) and the generous support from DST and other funding agencies, crystallographic research is at par with the rest of the world, both in small molecules and macro-molecular regime. As far as small molecule crystallography is concerned the focus has shifted completely towards understanding of chemical and biological molecules in terms of structure-function relations on one hand and generation of futuristic materials on the other. Expensive high quality machines are now accessible in every nook and corner of the country. Gautam Desiraju and I have played different roles (figure 10) to see that the efforts made by the two giants are

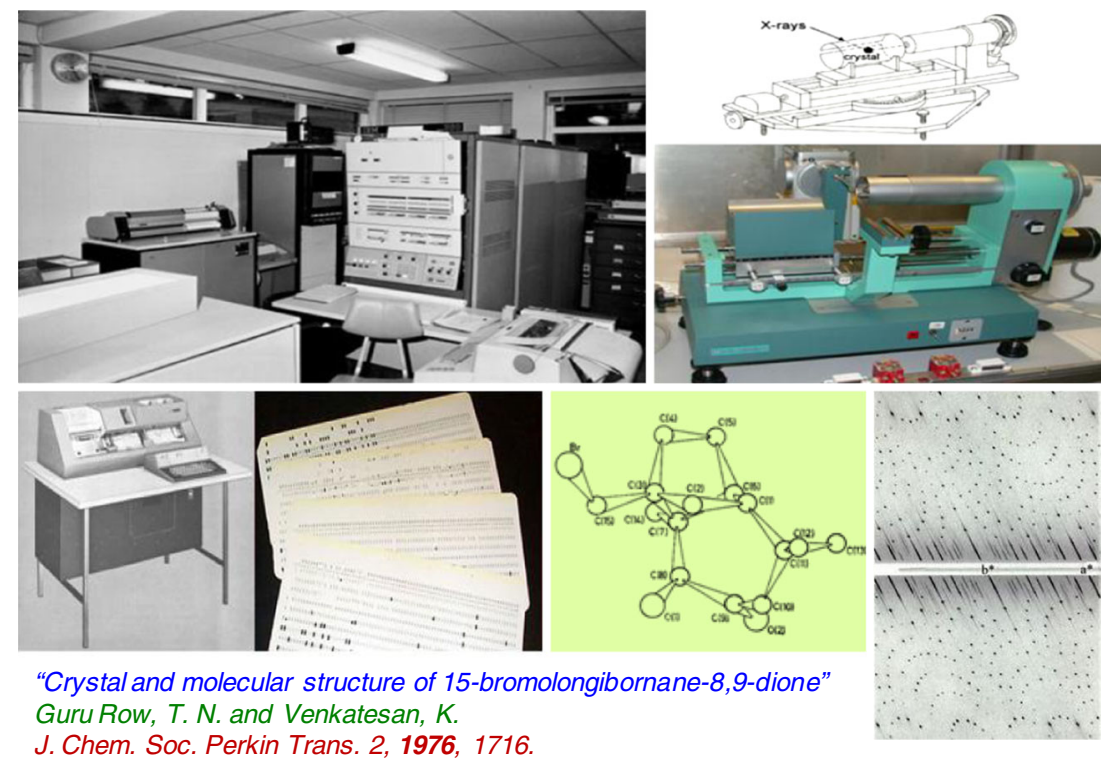

Figure 8. IBM360/44 computer, my first photograph from the Weissenberg camera and my first structure. 


\section{The Architects - Out of the Dark Age}

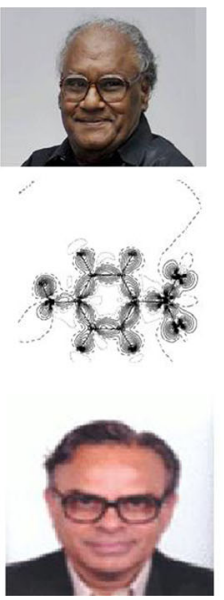

Quick to identify lacunae!

Establishment of facilities, both powder and single crystal modern day diffractometers, leading to powder diffraction activity, Rietveld refinements and ab initio structure determination.

Suggested to DST to set up nation-wide $X$-ray facilities.

The first charge -density analyses in India
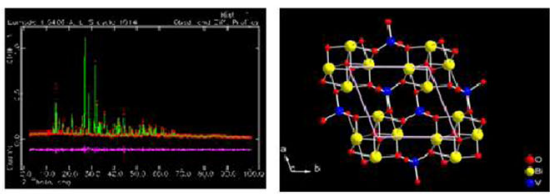

Made significant and major strides to develop structural biology in India. Set up the national facility for macromolecular data collection.

Procured state-of-the-art facilities and also assisted spread of the facilities (and faculty) to all parts of the country.

Ensured that the macromolecular crystallography (crystallographers) will produce highest level science in the country.

Some notable names : M. R. N. Murthy, S. Ramakumar, K. Suguna, T. P. Singh, D. Salunke, S. Mande, etc.

Figure 9. Crystallographic research was restored due to the efforts of C. N. R. Rao and Vijayan.

not wasted and some of the students of Vijayan (for example, T. P. Singh, Dinakar Salunke) have pitched in effectively.

The work done in the group of Dhanajai Pandey (BHU) in the area of ferric materials has been acknowledged in many international arenas. This group has kept the BHU's interest in the development of crystallographic research intact. There are several excellent groups, some of which are now in the forefront of crystallographic research in the country, figure 11. As can be seen from this small sample, which is by no means complete, crystallography in India is looking vibrant. Research in the pharmaceutical and other industry sectors is on the rise as can be seen from figure 11 as well. All these look
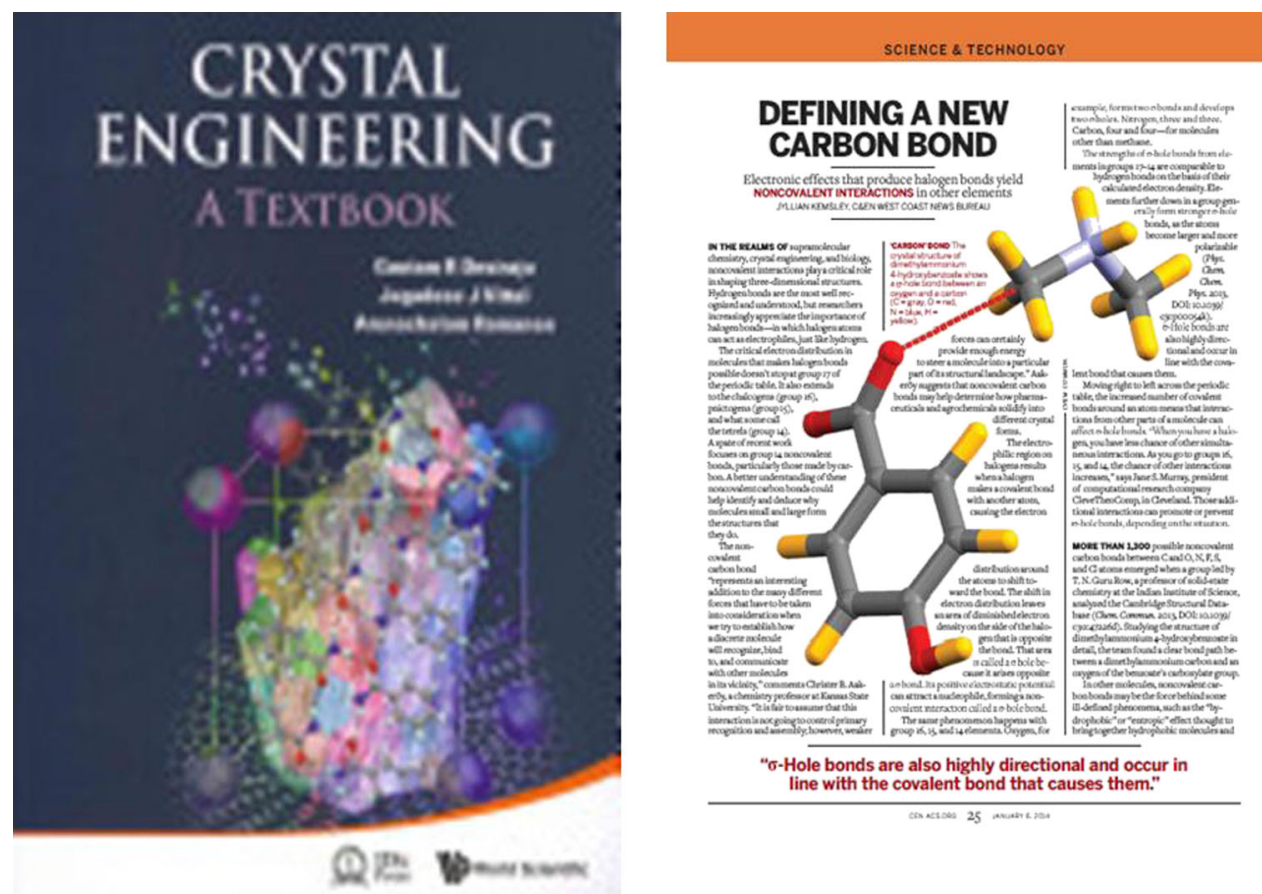

Figure 10. The current day expertise in small molecule crystallography and the contributions have been internationally acknowledged. 


\section{Current Status of Crystallographic Research}

- Macromolecular Crystallography

1. Structure based inhibitor/drug design: Inflammation (T. P. Singh)

2. Allergenic proteins (D. M. Salunke)

3. DNA repair system in M. Tuberculosis: Antibacterials (R. Ravishankar)

\section{Structure-Function Correlation Towards Design of Futuristic Drugs!}

Small molecule Crystallography

1. Functional supramolecular gelators (P. Dastidar)

2. Coordination polymers and MOFs (S. Natarajan, T. K. Maji and R. Banerjee)

3. Solid forms of API's: Polymorphism and cocrystal formation (A. Nangia)

\section{Futuristic Materials}

A significantly large number of scientists using crystallography!

\section{Year Wise Installation of Powder XRD and Single Crystal XRD from All Major Vendors}

\begin{tabular}{|ccc|}
\hline Year & Powder XRD $^{*}$ & $\begin{array}{c}\text { Single Crystal } \\
\text { XRD }\end{array}$ \\
\hline 2000 & 31 & 2 \\
\hline 2001 & 38 & 2 \\
\hline 2002 & 30 & 3 \\
\hline 2003 & 32 & 1 \\
\hline 2004 & 59 & 4 \\
\hline 2005 & 40 & 7 \\
\hline 2006 & 47 & 3 \\
\hline 2007 & 78 & 9 \\
\hline 2008 & 64 & 13 \\
\hline 2009 & 75 & 17 \\
\hline 2010 & 69 & 15 \\
\hline 2011 & 64 & 11 \\
\hline 2012 & 81 & 17 \\
\hline 2013 & 76 & 7 \\
\hline
\end{tabular}

*Powder XRD installations show the interests of the pharma industries!

Figure 11. A small sample of current day research groups in the country along with the number of X-ray diffractometers machines set up during the past 13 years.

very promising, however, the need to increase the number of researchers in crystallography and related areas along with the procurement of competitive experimental facilities (primarily Synchrotron) is becoming acute. Unless acted upon quickly, I perceive the advent of another serious dark age!

\section{The Future}

We are now involved in molecular property/solid state property correlations, crystal structure design and engineering, and theoretical studies, where, from the start, data and knowledge from X-ray 
structural studies marched side-by-side with the development and parameterisation of computational chemistry methods in molecular and solid state science. This combination is feeding back into crystal structure energy calculations, charge-density analysis, crystal structure prediction, and many aspects of material science and properties. - M. B. Hursthouse.

I must say that the views in the following sentences are entirely the way I see the future. It appears that a repeat 'dark age' is on the horizon if necessary actions are not taken. If one looks at the current literature, the areas pursued are modulated structures, disorder and reactivity, in situcryo crystallography, non-ambient crystallography and time resolved crystallography on one hand, and large proteins and enzymes (small crystals), complexes and structure based drug design, combining cognate techniques like NMR, electron diffraction (microscopy) and discerning time resolved biological processes on the other hand. It is also clearly seen that most of these areas are currently not pursued in India due to lack of facilities, though the expertise exists. Many young crystallographers, chemists, biologists and physicists are trained abroad and have returned and are egging to pursue work in some of these areas. The biggest hurdle is the non-availability of experimental facilities, in particular a state-of-theart synchrotron facility. These facilities, of course, are accessible for data collection through the generous support of DST, and it is indeed gratifying that most of the work is through collaboration with the experts in other countries and/or at synchrotron facilities. However, the number of researchers who would be able to access synchrotron facilities abroad is limited and nothing is better than having India's own centre. The facility at Indore is not state-of-the-art machine and resurrecting it would be like restructuring an old building. We certainly have the expertise in a large number of students, post-docs and engineers whom we have trained in India over the years and who have had a formal training and experience in operating the synchrotron facilities around the world. We just need to equip them to perform, and very soon the second Dark Age would come to an end.

\section{Conclusions}

This article is just an overview and there could be some small deviations in the narration with respect to time. I might have missed out some key persons and contributions to Indian Crystallography. I would like to acknowledge all my colleagues and seniors with whom I had discussions before I could come out with the contents herein.

September 2014

T. N. GURU ROW

Solid State and Structural Chemistry Unit Indian Institute of Science

Bangalore 560012, India

E-mail: ssctng@sscu.iisc.ernet.in 